\title{
ON THE RATE OF CONVERGENCE OF MOMENTS IN THE CENTRAL LIMIT THEOREM FOR LATTICE DISTRIBUTIONS
}

\author{
BY
}

\author{
PETER HALL
}

\begin{abstract}
We derive sharp asymptotic expressions for moments of the type $E\left\{b\left(\left|S_{n} / n^{1 / 2}\right|\right)\right\}$, where $S_{n}$ is a sum of independent lattice-valued random variables with finite variance, and $b$ is a concave function. It is shown that the behaviour of $b$ at the origin has a profound effect on the behaviour of such moments, and that this influence accounts for the major difference between the properties of moments of lattice and nonlattice sums. Asymptotic expansions for moments of sums of latticevalued variables are also derived.
\end{abstract}

1. Introduction. Let $X, X_{1}, X_{2}, \ldots$, be independent and identically distributed random variables with zero mean and unit variance, and set $S_{n}=\sum_{j=1}^{n} X_{j}$. Suppose the random variable $Z$ has the standard normal distribution. The central limit theorem for the partial sums $S_{n}$ holds in the form

$$
P\left(S_{n} / n^{1 / 2} \leqslant x\right) \rightarrow P(Z \leqslant x), \quad-\infty<x<\infty,
$$

and if $E|X|^{p}<\infty$ for some $p>0$ then $E\left|S_{n} / n^{1 / 2}\right|^{p} \rightarrow E|Z|^{p}$ as $n \rightarrow \infty$. (See Bernstein [2].) Results of Bhattacharya and Rao [3] can be used to derive a rate of convergence in this limit theorem for moments. For example, if $0<p<4, E\left(X^{4}\right)<$ $\infty$ and $X$ satisfies Cramér's continuity condition, i.e.

$$
\limsup _{t \rightarrow \infty}\left|E\left(e^{i t X}\right)\right|<\infty,
$$

then it follows from Theorem 20.1, p. 208 of [3] that

$$
E\left|S_{n} / n^{1 / 2}\right|^{p}=E|Z|^{p}+n^{-1} c_{p}+o\left(n^{-1}\right)
$$

as $n \rightarrow \infty$, where the constant $c_{p}$ depends only on $p$ and the first four moments of $X$. Earlier results of von Bahr [1] permit this result to be partially extended to lattice distributions. If $E|X|^{4+\varepsilon}<\infty$ for some $\varepsilon>0$, and if $p>1$, then (1.1) continues to hold even if the restriction (C) is dropped. (See Theorem 2 of [1].) In the borderline case $p=1$, (1.1) holds in the form $E\left|S_{n} / n^{1 / 2}\right|^{p}=E|Z|^{p}+O\left(n^{-1}\right)$. But fast rates of convergence of this type do not seem to be possible in the case $p<1$. Even if all the moments of $X$ are assumed to be finite, the best result permitted by von Bahr's Theorem 2 when $0<p<1$ is

$$
E\left|S_{n} / n^{1 / 2}\right|^{p}=E|Z|^{p}+O\left(n^{-(1 / 2)(p+1)}\right),
$$

Received by the editors February 19, 1982.

1980 Mathematics Subject Classification. Primary 60F05; Secondary 60G50.

Key words and phrases. Asymptotic expansion, lattice-valued variable, moments, rate of convergence. 
as $n \rightarrow \infty$. A similar result may be deduced from more recent studies of moment convergence [6,7]. It is clear from the result $(1.1)$ that this restriction on the rate of convergence has a lot to do with the behaviour of lattice distributions. Our aim in the present paper is to provide a deeper understanding of the properties of moments in the case of lattice distributions.

One corollary of our work is that if $X$ has a lattice distribution with maximal span $d$, and if $E\left(X^{4}\right)<\infty$, then there exists a (negative) constant $c_{p}^{\prime}$ depending only on $p$ and $d$, such that for $0<p<1$,

$$
E\left|S_{n} / n^{1 / 2}\right|^{p}=E|Z|^{p}+c_{p}^{\prime} n^{-(1 / 2)(p+1)}+o\left(n^{-(1 / 2)(p+1)}\right) .
$$

This result provides an analogue of (1.1) in the case of a lattice distribution. We actually study the more general problem of convergence of moments of the form $E\left\{b\left(\left|S_{n} / n^{1 / 2}\right|\right)\right\}$, where $b$ is a smooth function on $(0, \infty)$, such as $b(x) \equiv x^{p}$ or $b(x) \equiv x^{p}(\log x)^{m}$. This leads us to an interesting and unexpected conclusion: the term $c_{p}^{\prime} n^{-(1 / 2)(p+1)}$ in (1.3) is needed to correct $E\left|S_{n} / n^{1 / 2}\right|^{p}$ for errors close to the origin. The term can be dropped if we slightly alter our function $x^{p}$ so that it approaches the origin in a smooth way. For example, if $b(x)=x^{p}$ on $(1, \infty)$, and if $b$ is defined as $(0,1)$ in such a way that $b$ has two continuous derivatives on $[0,1]$ and

$$
\int_{0}^{1}\left|b^{\prime \prime}(x)\right| d x<\infty
$$

then

$$
E\left\{b\left(\left|S_{n} / n^{1 / 2}\right|\right)\right\}-E\{b(|Z|)\}=O\left(n^{-1}\right)
$$

as $n \rightarrow \infty$. The rate of convergence expressed by this result is a significant improvement over that described by (1.3). In general, if the concave function $b$ is constructed so that (1.4) holds, then the moments of partial sums from lattice and nonlattice distributions have the same first-order properties, up to terms of order $n^{-1}$.

During the discussion above it was convenient to assume that $E\left(X^{4}\right)<\infty$. In the great majority of the work below we do not require this condition, and our most severe moment restriction is that $X$ have finite variance. Thus, our results apply very generally. So as to compare lattice and nonlattice distributions we derive analogues of our main lattice results in the case of a smooth summand distribution. We also describe arbitrarily long asymptotic expansions for moments from a lattice distribution. All our results are presented together in $\$ 2$, and their proofs are deferred until $\S 3$.

2. Results. We adopt the notation of $\S 1$, and let $b$ be any function with two continuous derivatives on $(0, \infty)$ and satisfying

$$
\begin{gathered}
|b(x)|+x\left|b^{\prime}(x)\right| \rightarrow 0 \quad \text { as } x \rightarrow 0 \\
\int_{0}^{1}\left\{\left|b^{\prime}(x)\right|+x\left|b^{\prime \prime}(x)\right|\right\} d x<\infty \\
\int_{1}^{\infty}\left\{x^{-2}|b(x)|+x^{-1}\left|b^{\prime}(x)\right|+\left|b^{\prime \prime}(x)\right|\right\} d x<\infty
\end{gathered}
$$

and

$$
b^{\prime \prime}(x) \text { does not change sign in some interval }(0, \varepsilon), \varepsilon>0
$$


For example, we may take $b(x) \equiv x^{p}$ for any $p \in(0,1)$. It follows from Lemma 1 and the result (3.4) in $\S 3$ that under conditions (2.1)-(2.3), the function

$$
\begin{aligned}
\beta(t) & \equiv-(2 / \pi)(d / d t) \int_{0}^{\infty} x^{-1} b(x) \sin (t x) d x \\
& =-\left(2 / \pi t^{2}\right) \int_{0}^{\infty} b^{\prime \prime}(x)(1-\cos t x) d x
\end{aligned}
$$

is well defined.

Set $\delta_{n}=E\left\{X^{2} I\left(|X|>n^{1 / 2}\right)\right\}+n^{-1} E\left\{X^{4} I\left(|X| \leqslant n^{1 / 2}\right)\right\}$, where $I(E)$ denotes the indicator function of the event $E$. We consider first the case of a lattice distribution.

THEOREM 1. If $X$ has a lattice distribution with span $d$ then

$$
\begin{aligned}
E\left\{b\left(\left|S_{n} / n^{1 / 2}\right|\right)\right\} & -E\{b(|Z|)\} \\
= & -n \int_{0}^{\infty} \beta(t) E\left\{\cos \left(t X / n^{1 / 2}\right)-1+\frac{1}{2}\left(t X / n^{1 / 2}\right)^{2}\right\} e^{-t^{2} / 2} d t \\
& -r_{n}+o\left(\delta_{n}\right)+O\left(n^{-1}\right)
\end{aligned}
$$

as $n \rightarrow \infty$, where

$$
r_{n}=\{1+o(1)\}(2 \pi)^{1 / 2} \sum_{j=1}^{\infty} \beta\left(2 j \pi n^{1 / 2} / d\right) .
$$

We shall comment first on the role of the term $o\left(\delta_{n}\right)$ in (2.6). Let us suppose that the function $\beta$ does not change sign on $(0, \infty)$. A sufficient condition for this property is that $b$ be concave on $(0, \infty)$ (e.g. $b(x) \equiv x^{p}, 0<p<1$ ), for then $b^{\prime \prime}(x) \leqslant 0$ and the last equality in (2.5) implies that $\beta(t)>0$ on $(0, \infty)$. Theorem 2 below shows that in these circumstances, the term $o\left(\delta_{n}\right)$ is negligible in comparison with the first term on the right in (2.6).

THEOREM 2. If $\beta$ does not change sign on $(0, \infty)$, then there exist positive constants $C_{1}$ and $C_{2}$ such that

$$
C_{1} \delta_{n}<n\left|\int_{0}^{\infty} \beta(t) E\left\{\cos \left(t X / n^{1 / 2}\right)-1+\frac{1}{2}\left(t X / n^{1 / 2}\right)^{2}\right\} e^{-t^{2} / 2} d t\right| \leqslant C_{2} \delta_{n}
$$

for all $n$.

Note that Theorem 2 holds for both lattice and nonlattice distributions. If the distribution of $X$ is lattice, and if in addition to the conditions (2.1)-(2.3), $b$ is concave, then the result (2.6) may be simplified to

$$
\begin{aligned}
E\left\{b\left(\left|S_{n} / n^{1 / 2}\right|\right)\right\}-E\{b(|Z|)\} & \\
= & -\{1+o(1)\} n \int_{0}^{\infty}\left|\beta(t) E\left\{\cos \left(t X / n^{1 / 2}\right)-1+\frac{1}{2}\left(t X / n^{1 / 2}\right)^{2}\right\}\right| e^{-t^{2} / 2} d t \\
& -\{1+o(1)\}(2 \pi)^{1 / 2} \sum_{j=1}^{\infty}\left|\beta\left(2 j \pi n^{1 / 2} / d\right)\right|+O\left(n^{-1}\right),
\end{aligned}
$$

as $n \rightarrow \infty$. 
Next we examine the term $r_{n}$ in (2.6). If the function $b^{\prime \prime}$ satisfies

$$
\int_{0}^{1}\left|b^{\prime \prime}(x)\right| d x<\infty
$$

then we may deduce from the last equality in (2.5) that $\beta(t)=O\left(t^{-2}\right)$ as $t \rightarrow \infty$, and it is clear from (2.7) that $r_{n}=O\left(n^{-1}\right)$ as $n \rightarrow \infty$. In this case the term $r_{n}$ may be dropped from (2.6), and the series may be dropped from (2.9). Thus, we may deduce from (2.9) that if $b$ is concave, the difference $E\left\{b\left(\left|S_{n} / n^{1 / 2}\right|\right)\right\}-E\{b(|Z|)\}$ is negative and of precise order $\delta_{n}$, up to terms of order $n^{-1}$. Therefore the quantity $E\{b(|Z|)\}$ provides an overestimate of $E\left\{b\left(\left|S_{n} / n^{1 / 2}\right|\right)\right\}$, up to terms of order $n^{-1}$. If $E\left(X^{4}\right)=\infty$ then $\delta_{n}$ will dominate $n^{-1}$ as $n \rightarrow \infty$.

It is important to note that condition (2.10) depends only on the behaviour of $b$ in the neighbourhood of the origin. A slight modification to $b$ on any interval $(0, \varepsilon)$, retaining concavity if $b$ had that property, will ensure that (2.10) holds and permit the term $r_{n}$ to be dropped from (2.6). However, $r_{n}$ must be retained in several important cases. Consider for example the case $b(x) \equiv x^{p}$, where $0<p<1$. Then

$$
\beta(t)=-2 p(p-1) \pi^{-1} t^{-(p+1)} \int_{0}^{\infty} x^{p-2}(1-\cos x) d x=K_{p} t^{-(p+1)},
$$

say, where $K_{p}=2 \Gamma(p+1) \sin (p \pi / 2) / \pi>0$. Consequently

$$
\begin{aligned}
r_{n} & \sim(2 \pi)^{1 / 2} \sum_{j=1}^{\infty}\left|\beta\left(2 j \pi n^{1 / 2} / d\right)\right|=d^{p+1}\left\{\left(\sum_{1}^{\infty} j^{-(p+1)}\right) K_{p} /(2 \pi)^{p+(1 / 2)}\right\} n^{-(p+1) / 2} \\
& =d^{p+1} L_{p} n^{-((1 / 2)+1) / 2},
\end{aligned}
$$

say. We may now deduce from Theorem 2 that the quantity $r_{n}$ will dominate the other terms in (2.6) if and only if $\delta_{n}=o\left(n^{-(p+1) / 2}\right)$. This leads to the following result.

THEOREM 3. If the random variable $X$ has a lattice distribution with maximal span $d$, and if $0<p<1$, then

$$
E\left(\left|S_{n} / n^{1 / 2}\right|^{p}\right)-E\left(|Z|^{p}\right)=-d^{p+1} L_{p} n^{-(p+1) / 2}+o\left(n^{-(p+1) / 2}\right)
$$

if and only if

$$
x^{p+3} P(|X|>x) \rightarrow 0
$$

as $x \rightarrow \infty$.

Note that (2.11) is implied by the moment condition $E\left(|X|^{p+3}\right)<\infty$, and implies that $E\left(|X|^{p+3-\varepsilon}\right)<\infty$ for each small $\varepsilon>0$.

By way of comparison with the preceding work, we shall briefly consider the case of a nonlattice distribution. Our first result is an analogue of Theorem 1.

THEOREM 4. If the distribution of $X$ satisfies Cramér's continuity condition $(\mathrm{C})$ (see \$1) then

$$
\begin{aligned}
& E\left\{b\left(\left|S_{n} / n^{1 / 2}\right|\right)\right\}-E\{b(|Z|)\} \\
& \quad=-n \int_{0}^{\infty} \beta(t) E\left\{\cos \left(t X / n^{1 / 2}\right)-1+\frac{1}{2}\left(t X / n^{1 / 2}\right)^{2}\right\} e^{-t^{2} / 2} d t+o\left(\delta_{n}\right)+O\left(n^{-1}\right)
\end{aligned}
$$

as $n \rightarrow \infty$. 
Of course, not all nonlattice distributions satisfy Cramér's continuity condition. Bhattacharya and Rao [3, p. 207] discuss the implications of condition (C). In order to reduce the gap between lattice distributions and those distributions satisfying $(\mathrm{C})$, we consider a refinement of Theorem 4 in the special case $b(x) \equiv x^{p}$.

THEOREM 5. Suppose the characteristic function of $X$ satisfies the condition

$$
\liminf _{t \rightarrow \infty} t^{2 p /(2-p)}\left\{1-\left|E\left(e^{i t X}\right)\right|\right\}>0,
$$

where $0<p<1$. Then

$$
\begin{aligned}
E\left(\left|S_{n} / n^{1 / 2}\right|^{p}\right)-E\left(|Z|^{p}\right) & \\
= & -\{1+o(1)\} K_{p} n \int_{0}^{\infty} t^{-(p+1)} E\left\{\cos \left(t X / n^{1 / 2}\right)-1+\frac{1}{2}\left(t X / n^{1 / 2}\right)^{2}\right\} \\
& \times e^{-t^{2} / 2} d t+O\left(n^{-1}\right)
\end{aligned}
$$

as $n \rightarrow \infty$.

Note that condition (C) implies (2.12).

We introduced our discussion of moment properties by considering a short asymptotic expansion in the case of a smooth summand distribution (see (1.1)). We shall conclude with an asymptotic expansion for lattice distributions. Let us assume that $E\left(X^{2 k}\right)<\infty$ for an integer $k \geqslant 2$, and let $\mu_{j}=E\left(X^{j}\right)$ for $1 \leqslant j \leqslant 2 k$. We adopt von Bahr's [1] notation, in which $P_{l}(t)$ is a polynomial in $t$ of degree $3 l$ whose coefficients depend only on $\mu_{1}, \ldots, \mu_{l+2}$. Note that $P_{l}$ is an odd function if $l$ is odd, and an even function if $l$ is even. Let $(\underset{m}{-(p+1)})$ stand for the negative binomial coefficient.

THEOREM 6. If $X$ has a lattice distribution with maximal span $d$, if $E\left(X^{2 k}\right)<\infty$ for an integer $k \geqslant 2$ and if $0<p<2 k$, then

$$
\begin{aligned}
& E\left(\left|S_{n} / n^{1 / 2}\right|^{p}\right)-E\left(|Z|^{p}\right)=\sum_{j=1}^{k-1} n^{-j} \int_{-\infty}^{\infty}|x|^{p} d P_{2 j}(-\Phi)(x) \\
& -K_{p} \sum_{l=0}^{[k-1-(1 / 2)(p+1)][k-l-1-(1 / 2)(p+1)]} \sum_{m=0} n^{-l-m-(1 / 2)(p+1)} s_{2 m+p+1}\left(\begin{array}{c}
-(p+1) \\
2 m
\end{array}\right) \\
& \times(d / 2 \pi)^{2 m+p+1} \int_{-\infty}^{\infty} t^{2 m} P_{2 l}(i t) e^{-t^{2} / 2} d t+o\left(n^{1-k}\right)
\end{aligned}
$$

In (2.13), $i=\sqrt{-1}$ and $[x]$ equals the largest integer not exceeding $x$. Note that $P_{2 l}(i t)$ is a real-valued function of $t$. In the case where $p$ is an even integer, $K_{p}=0$.

In the case of smooth functions $b$, Edgeworth expansions are available in the general case. See for example [3, Theorem 20.7] and [5].

3. Proofs. The following lemma is the key to our estimation procedure. It provides a Fourier transform and an inversion formula for a special class of functions. 
LEMMA 1. If conditions (2.1), (2.2) and (2.3) hold then the function

$$
\beta(t)=-(2 / \pi)(d / d t) \int_{0}^{\infty} x^{-1} b(x) \sin (t x) d x
$$

is well defined on $(0, \infty)$, bounded on intervals of the form $(\varepsilon, \infty)$ for $\varepsilon>0$, and satisfies

$$
\int_{0}^{\infty}|\beta(t)| \min \left(t^{2}, 1\right) d t<\infty .
$$

Furthermore,

$$
\int_{0}^{\infty} \beta(t)(1-\cos t x) d t \equiv b(x), \quad x>0 .
$$

The Fourier transform of $|x|^{p}$ may be found in [4, p. 359].

Proof. Observe first that

$$
x^{-1} b(x)=\int\left\{x^{-1} b^{\prime}(x)-x^{-2} b(x)\right\} d x+\text { const. }
$$

and so the existence of $\lim _{x \rightarrow \infty} x^{-1} b(x)$ follows from (2.3). If the limit is nonzero, this will force $\int_{1}^{\infty} x^{-2}|b(x)| d x=\infty$, contradicting (2.3). Therefore

$$
\lim _{x \rightarrow \infty} x^{-1} b(x)=0 \text {. }
$$

Now for any $\lambda, t>0$,

$$
\begin{array}{rl}
\int_{0}^{\lambda} x^{-1} & b(x) \sin (t x) d x \\
& =t^{-1} \lambda^{-1} b(\lambda)(1-\cos t \lambda)+t^{-1} \int_{0}^{\lambda}\left\{x^{-2} b(x)-x^{-1} b^{\prime}(x)\right\}(1-\cos t x) d x \\
& \rightarrow t^{-1} \int_{0}^{\infty}\left\{x^{-2} b(x)-x^{-1} b^{\prime}(x)\right\}(1-\cos t x) d x \\
& =\int_{0}^{\infty}\left\{x^{-2} b(x / t)-x^{-1} t^{-1} b^{\prime}(x / t)\right\}(1-\cos x) d x
\end{array}
$$

as $\lambda \rightarrow \infty$, using (2.3) and (3.3). The latter integral is absolutely convergent, and on differentiating with respect to $t$ under the integral sign we find that

$$
\begin{aligned}
(d / d t) \int_{0}^{\infty} x^{-1} b(x) \sin (t x) d x & =t^{-3} \int_{0}^{\infty} b^{\prime \prime}(x / t)(1-\cos x) d x \\
& =t^{-2} \int_{0}^{\infty} b^{\prime \prime}(x)(1-\cos t x) d x=\beta_{1}(t),
\end{aligned}
$$

say. The second-written integral converges uniformly and the integrand is continuous in both variables, and so the differentiation was justified.

The boundedness of $\beta_{1}$ (and so of $\beta$ ) on $(\varepsilon, \infty)$ follows from the fact that

$$
\left|\beta_{1}(t)\right| \leqslant \int_{0}^{1} x^{2}\left|b^{\prime \prime}(x)\right| d x+2 t^{-2} \int_{1}^{\infty}\left|b^{\prime \prime}(x)\right| d x .
$$

To prove (3.1), observe that

$$
\begin{aligned}
\int_{0}^{\infty}\left|\beta_{1}(t)\right| \min \left(t^{2}, 1\right) d t & \leqslant \int_{0}^{\infty}\left|b^{\prime \prime}(x)\right| d x \int_{0}^{\infty} \min \left(1, t^{-2}\right)(1-\cos t x) d t \\
& \leqslant C \int_{0}^{\infty}\left|b^{\prime \prime}(x)\right| \min (x, 1) d x<\infty
\end{aligned}
$$


Consequently

$$
\int_{0}^{\infty} \beta_{1}(t)(1-\cos t z) d t=\int_{0}^{\infty} b^{\prime \prime}(x) d x \int_{0}^{\infty} t^{-2}(1-\cos t x)(1-\cos t z) d t
$$

for all $z>0$. But

$$
\begin{aligned}
\int_{0}^{\infty} t^{-2}(1-\cos t x)(1-\cos t z) d t \\
=\int_{0}^{\infty} t^{-1}\{x \sin (t x)(1-\cos t z)+z \sin (t z)(1-\cos t x)\} d t \\
=(\pi / 2) x\left[\operatorname{sgn}(x)-\frac{1}{2}\{\operatorname{sgn}(x+z)+\operatorname{sgn}(x-z)\}\right] \\
\quad+(\pi / 2) z\left[\operatorname{sgn}(z)-\frac{1}{2}\{\operatorname{sgn}(z+x)+\operatorname{sgn}(z-x)\}\right] \\
=(\pi / 2) \min (x, z) .
\end{aligned}
$$

Therefore

$$
(2 / \pi) \int_{0}^{\infty} \beta_{1}(t)(1-\cos t z) d t=\int_{0}^{z} x b^{\prime \prime}(x) d x+z \int_{z}^{\infty} b^{\prime \prime}(x) d x=-b(z),
$$

which proves (3.2).

Proof of TheOREMS 1, 4 AND 5. Let $\alpha$ be the common characteristic function of the summands $X_{j}$. It follows directly from (3.2) that

$$
E\left\{b\left(S_{n} / n^{1 / 2}\right)-b(Z)\right\}=-\int_{0}^{\infty} \beta(t)\left\{\operatorname{Re} \alpha^{n}\left(t / n^{1 / 2}\right)-e^{-t^{2} / 2}\right\} d t
$$

We shall break the integral on the right into two parts,

$$
\int_{0}^{\infty}=\int_{0}^{\varepsilon n^{1 / 2}}+\int_{\varepsilon n^{1 / 2}}^{\infty},
$$

where $\varepsilon$ is a small positive number. In our handling of the first part we draw no distinction between lattice and nonlattice distributions.

Since $\log \alpha(t)=\alpha(t)-1+O\left(t^{4}\right)$ as $t \rightarrow 0$ then

$$
\begin{aligned}
\alpha^{n}\left(t / n^{1 / 2}\right) & =e^{-t^{2} / 2} \exp \left[n\left\{\alpha\left(t / n^{1 / 2}\right)-1+\frac{1}{2}\left(t / n^{1 / 2}\right)^{2}\right\}+r_{n 1}(t)\right] \\
& =e^{-t^{2} / 2}\left[1+n\left\{\alpha\left(t / n^{1 / 2}\right)-1+\frac{1}{2}\left(t / n^{1 / 2}\right)^{2}\right\}+r_{n 1}(t)+r_{n 2}(t)\right],
\end{aligned}
$$

where $\left|r_{n 1}(t)\right| \leqslant C n^{-1} t^{4}$ and

$$
\begin{aligned}
\left|r_{n 2}(t)\right| \leqslant & 2\left\{n^{2}\left|\alpha\left(t / n^{1 / 2}\right)-1+\frac{1}{2}\left(t / n^{1 / 2}\right)^{2}\right|^{2}+\left|r_{n 1}(t)\right|^{2}\right\} \\
& \times \exp \left\{n\left|\alpha\left(t / n^{1 / 2}\right)-1+\frac{1}{2}\left(t / n^{1 / 2}\right)^{2}\right|+\left|r_{n 1}(t)\right|\right\}
\end{aligned}
$$




$$
\begin{aligned}
& \text { for }|t| \leqslant n^{1 / 2} \text {. But } \alpha(t)-1+\frac{1}{2} t^{2}=o\left(t^{2}\right) \text { as } t \rightarrow 0, \text { and } \\
& \begin{aligned}
n\left|\alpha\left(t / n^{1 / 2}\right)-1+\frac{1}{2}\left(t / n^{1 / 2}\right)^{2}\right| \\
=n \mid E\left\{\cos \left(t X / n^{1 / 2}\right)-1+\frac{1}{2}\left(t X / n^{1 / 2}\right)^{2}\right\}
\end{aligned} \\
& +i E\left[\left\{\sin \left(t X / n^{1 / 2}\right)-\left(t X / n^{1 / 2}\right)\right\} I\left(|X|>n^{1 / 2}\right)\right] \\
& +i E\left[\left\{\sin \left(t X / n^{1 / 2}\right)-\left(t X / n^{1 / 2}\right)+\frac{1}{6}\left(t X / n^{1 / 2}\right)^{3}\right\} I\left(|X| \leqslant n^{1 / 2}\right)\right] \\
& \quad-i E\left\{\frac{1}{6}\left(t X / n^{1 / 2}\right)^{3} I\left(|X| \leqslant n^{1 / 2}\right)\right\} \mid \\
& \leqslant 4\left(t^{2}+t^{4}\right)\left[E\left\{X^{2} I\left(|X|>n^{1 / 2}\right)\right\}+n^{-1} E\left\{X^{4} I\left(|X| \leqslant n^{1 / 2}\right)\right\}\right. \\
& \left.\quad+n^{-1 / 2}\left|E\left\{X^{3} I\left(|X| \leqslant n^{1 / 2}\right)\right\}\right|\right] .
\end{aligned}
$$

Therefore if $\varepsilon$ is chosen sufficiently small,

$$
\begin{aligned}
\left|r_{n 2}(t)\right| \leqslant C\left(t^{4}+t^{8}\right)\left[E\left\{X^{2} I\left(|X|>n^{1 / 2}\right)\right\}\right. & +n^{-1} E\left\{X^{4} I\left(|X| \leqslant n^{1 / 2}\right)\right\} \\
& \left.+n^{-1 / 2}\left|E\left\{X^{3} I\left(|X| \leqslant n^{1 / 2}\right)\right\}\right|\right]^{2} e^{t^{2} / 4}
\end{aligned}
$$

for all large $n$ and all $|t| \leqslant \varepsilon n^{1 / 2}$. It is shown in Lemma 2 of [7] that

$$
n^{-1}\left|E\left\{X^{3} I\left(|X| \leqslant n^{1 / 2}\right)\right\}\right|^{2}=O\left(n^{-1}\right)+o\left[n^{-1} E\left\{X^{4} I\left(|X| \leqslant n^{1 / 2}\right)\right\}\right]
$$

as $n \rightarrow \infty$, and so the square of the term within square brackets in (3.8) equals $o\left(\delta_{n}\right)+O\left(n^{-1}\right)$. When this estimate is substituted into (3.7) we may deduce that

$$
\begin{aligned}
& \int_{0}^{\varepsilon n^{1 / 2}} \beta(t)\left\{\operatorname{Re} \alpha^{n}\left(t / n^{1 / 2}\right)-e^{-t^{2} / 2}\right\} d t \\
&=\int_{0}^{\varepsilon n^{1 / 2}} \beta(t) n E\left\{\cos \left(t X / n^{1 / 2}\right)-1+\frac{1}{2}\left(t X / n^{1 / 2}\right)^{2}\right\} e^{-t^{2} / 2} d t \\
&+o\left(\delta_{n}\right)+O\left(n^{-1}\right) \\
&= n \int_{0}^{\infty} \beta(t) E\left\{\cos \left(t X / n^{1 / 2}\right)-1+\frac{1}{2}\left(t X / n^{1 / 2}\right)^{2}\right\} e^{-t^{2} / 2} d t \\
&+o\left(\delta_{n}\right)+O\left(n^{-1}\right) .
\end{aligned}
$$

(Note the properties of $\beta$ derived in Lemma 1.)

If $\alpha$ satisfies condition (C) then the second integral on the right in (3.6) is dominated by

$$
\left[\left\{\sup _{t>\varepsilon}|\alpha(t)|\right\}^{n}+e^{-\varepsilon^{2} n / 2}\right] \int_{\varepsilon n^{1 / 2}}^{\infty}|\beta(t)| d t=O\left(n^{-k}\right)
$$

for all $k>0$. Theorem 4 follows on combining this estimate with (3.5) and (3.9). In the special case $b(x) \equiv x^{p}$ of Theorem 5, we estimate the second integral in (3.6) as 
follows. Writing

$$
\delta=\inf _{t>\varepsilon} t^{2 p /(2-p)}\{1-|\alpha(t)|\}>0
$$

we have

$$
\begin{gathered}
\int_{\varepsilon n^{1 / 2}}^{\infty}|\beta(t)|\left|\alpha\left(t / n^{1 / 2}\right)\right|^{n} d t \leqslant n^{1 / 2} \int_{\varepsilon}^{\infty}\left|\beta\left(n^{1 / 2} t\right)\right| \exp [-n\{1-|\alpha(t)|\}] d t \\
\leqslant K_{p} n^{-p / 2} \int_{\varepsilon}^{\infty} t^{-(p+1)} \exp \left(-n \delta / t^{2 p /(2-p)}\right) d t \\
=K_{p}\left(p^{-1}-(1 / 2)\right) n^{-1} \int_{0}^{n / \varepsilon^{k}} u^{-p / 2} e^{-\delta u} d u=O\left(n^{-1}\right),
\end{gathered}
$$

on changing variable to $u=n / t^{2 p /(2-p)}$. Theorem 5 now follows from (3.5) and (3.9).

To prove Theorem 1 we observe that when $X$ is lattice with span $d$, the characteristic function $\alpha$ is periodic with period $2 \pi / d$. We may suppose that $\varepsilon<\pi / d$, in which case

$$
\left|\int_{\varepsilon n^{1 / 2}}^{\pi n^{1 / 2} / d} \beta(t)\left\{\operatorname{Re} \alpha^{n}\left(t / n^{1 / 2}\right)-e^{-t^{2} / 2}\right\} d t-\int_{\pi n^{1 / 2} / d}^{\infty} \beta(t) e^{-t^{2} / 2} d t\right|=O\left(n^{-k}\right)
$$

for all $k>0$, since $\int_{1}^{\infty}|\beta(t)| d t<\infty$ and $\sup _{\varepsilon<t<\pi / d}|\alpha(t)|<1$. Therefore, rather than handle the second integral in (3.6), we may examine instead

$$
\begin{aligned}
I_{n} & =\int_{\pi n^{1 / 2} / d}^{\infty} \beta(t) \operatorname{Re} \alpha^{n}\left(t / n^{1 / 2}\right) d t \\
& =\sum_{j=1}^{\infty} \int_{-\pi n^{1 / 2} / d}^{\pi n^{1 / 2} / d} \beta\left(t+2 j \pi n^{1 / 2} / d\right) \operatorname{Re} \alpha^{n}\left(t / n^{1 / 2}\right) d t .
\end{aligned}
$$

The difference between this series and

$$
\sum_{j=1}^{\infty} \int_{-\varepsilon n^{1 / 2}}^{\varepsilon n^{1 / 2}} \beta\left(t+2 j \pi n^{1 / 2} / d\right) \operatorname{Re} \alpha^{n}\left(t / n^{1 / 2}\right) d t
$$

is dominated by

$$
\left\{\sup _{\varepsilon<t<\pi / d}|\alpha(t)|\right\}^{n} \int_{\pi n^{1 / 2} / d}^{\infty}|\beta(t)| d t=O\left(n^{-k}\right)
$$

for all $k>0$. Furthermore, the estimates (3.7) and (3.8) show that

$$
\begin{gathered}
\alpha^{n}\left(t / n^{1 / 2}\right)=e^{-t^{2} / 2}\left\{1+n \gamma\left(t / n^{1 / 2}\right)+r_{n 3}(t)\right\}, \\
\text { where } \gamma(t)=o\left(t^{2}\right) \text { as } t \rightarrow 0 \text { and }\left|r_{n 3}(t)\right| \leqslant o(1)\left(t^{4}+t^{8}\right) e^{t^{2} / 4} \text { for }|t| \leqslant \varepsilon n^{1 / 2} \text {. Since } \\
\sum_{j=1}^{\infty} \int_{-\varepsilon n^{1 / 2}}^{\varepsilon n^{1 / 2}}\left|\beta\left(t+2 j \pi n^{1 / 2} / d\right)\right|\left\{n\left|\gamma\left(t / n^{1 / 2}\right)\right|+\left|r_{n 3}(t)\right|\right\} e^{-t^{2} / 2} d t \\
\leqslant o(1) \sum_{j=1}^{\infty} \int_{-\varepsilon n^{1 / 2}}^{\varepsilon n^{1 / 2}}\left|\beta\left(t+2 j \pi n^{1 / 2} / d\right)\right| e^{-t^{2} / 8} d t
\end{gathered}
$$


then the latter quantity dominates the difference between the series in (3.12) and the series

$$
\sum_{j=1}^{\infty} \int_{-\varepsilon n^{1 / 2}}^{\varepsilon n^{1 / 2}} \beta\left(t+2 j \pi n^{1 / 2} / d\right) e^{-t^{2} / 2} d t .
$$

Combining the estimates from (3.11) down, we find that

$$
\begin{aligned}
I_{n}= & \sum_{j=1}^{\infty} \int_{-\varepsilon n^{1 / 2}}^{\varepsilon n^{1 / 2}} \beta\left(t+2 j \pi n^{1 / 2} / d\right) e^{-t^{2} / 2} d t \\
& +o(1) \sum_{j=1}^{\infty} \int_{-\varepsilon n^{1 / 2}}^{\varepsilon n^{1 / 2}}\left|\beta\left(t+2 j \pi n^{1 / 2} / d\right)\right| e^{-t^{2} / 8} d t+O\left(n^{-1}\right) .
\end{aligned}
$$

Choose the positive number $\zeta$ so small that $b^{\prime \prime}$ does not change sign on $(0, \zeta)$ (note condition (2.4)), and observe that with

$$
\beta_{2}(t) \equiv-(2 / \pi) t^{-2} \int_{0}^{\zeta} b^{\prime \prime}(x)(1-\cos t x) d x
$$

we have $\beta(t)=\beta_{2}(t)+O\left(t^{-2}\right)$ as $t \rightarrow \infty$. Therefore

$$
\begin{aligned}
\sum_{j=1}^{\infty} \int_{-\varepsilon n^{1 / 2}}^{\varepsilon n^{1 / 2}} \beta\left(t+2 j \pi n^{1 / 2} / d\right) e^{-t^{2} / 2} d t \\
=\sum_{j=1}^{\infty} \int_{-\varepsilon n^{1 / 2}}^{\varepsilon n^{1 / 2}} \beta_{2}\left(t+2 j \pi n^{1 / 2} / d\right) e^{-t^{2} / 2} d t+O\left(n^{-1}\right) .
\end{aligned}
$$

Next observe that

$$
(\pi / 2) t^{2} \beta_{2}(t)=\imath \int_{0}^{\zeta} b^{\prime}(x) \sin t x d x-b^{\prime}(\zeta)(1-\cos t \zeta)
$$

and so in view of condition (2.2), $\beta_{2}(t)=O\left(t^{-1}\right)$ as $t \rightarrow \infty$. Consequently

$$
\beta_{2}^{\prime}(t)=-(2 / \pi) t^{-2} \int_{0}^{\zeta} x b^{\prime \prime}(x) \sin t x d x-2 t^{-1} \beta_{2}(t)=O\left(t^{-2}\right)
$$

as $t \rightarrow \infty$. (Note condition (2.2).) Hence if $|t| \leqslant \varepsilon n^{1 / 2}$,

$$
\begin{aligned}
& \left|\beta_{2}\left(t+2 j \pi n^{1 / 2} / d\right)-\beta_{2}\left(2 j \pi n^{1 / 2} / d\right)\right| \\
& \leqslant|t| \sup _{\left|s-2 j \pi n^{1 / 2} / d\right| \leqslant \varepsilon n^{1 / 2}}\left|\beta_{2}^{\prime}(s)\right| \leqslant C n^{-1} j^{-2}|t|,
\end{aligned}
$$

where $C$ does not depend on $n, j$ or $t$. It follows that

$$
\begin{aligned}
& \sum_{j=1}^{\infty} \int_{-\varepsilon n^{1 / 2}}^{n^{1 / 2}} \beta_{2}\left(t+2 j \pi n^{1 / 2} / d\right) e^{-t^{2} / 2} d t \\
&=\left\{\sum_{j=1}^{\infty} \beta_{2}\left(2 j \pi n^{1 / 2} / d\right)\right\} \int_{-\varepsilon n^{1 / 2}}^{\varepsilon n^{1 / 2}} e^{-t^{2} / 2} d t+O\left(n^{-1}\right) .
\end{aligned}
$$


A similar argument shows that

$$
\begin{aligned}
& \sum_{j=1}^{\infty} \int_{-\varepsilon n^{1 / 2}}^{\varepsilon n^{1 / 2}}\left|\beta\left(t+2 j \pi n^{1 / 2} / d\right)\right| \exp \left(-\frac{1}{2} C_{1} t^{2}\right) d t \\
& =O\left\{\sum_{j=1}^{\infty}\left|\beta_{2}\left(2 j \pi n^{1 / 2} / d\right)\right|\right\}=O\left\{\left|\sum_{j=1}^{\infty} \beta_{2}\left(2 j \pi n^{1 / 2} / d\right)\right|\right\} .
\end{aligned}
$$

(Note that by the definition of $\beta_{2}$, that function is of the one sign for all $t>0$.) Combining (3.13)-(3.16) we find that

$$
\begin{aligned}
I_{n} & =\{1+o(1)\}(2 \pi)^{1 / 2} \sum_{j=1}^{\infty} \beta_{2}\left(2 j \pi n^{1 / 2} / d\right)+O\left(n^{-1}\right) \\
& =\{1+o(1)\}(2 \pi)^{1 / 2} \sum_{j=1}^{\infty} \beta\left(2 j \pi n^{1 / 2} / d\right)+O\left(n^{-1}\right) .
\end{aligned}
$$

Theorem 1 follows on combining this estimate with (3.5), (3.9), (3.10) and (3.11).

Proof of Theorem 2. There exist positive constants $C_{1}$ and $C_{2}$ such that

$$
C_{1} x^{2} \min \left(x^{2}, 1\right) \leqslant \cos x-1+\frac{1}{2} x^{2} \leqslant C_{2} x^{2} \min \left(x^{2}, 1\right)
$$

for all $x$, and so the absolute value of the integral in (2.8) dominates and is dominated by constant multiples of the quantity

$$
n E\left\{\int_{0}^{n^{1 / 2} /|X|}\left(t X / n^{1 / 2}\right)^{4}|\beta(t)| e^{-t^{2} / 2} d t+\int_{n^{1 / 2} /|X|}^{\infty}\left(t X / n^{1 / 2}\right)^{2}|\beta(t)| e^{-t^{2} / 2} d t\right\} .
$$

This in turn can be shown to dominate and be dominated by constant multiples of $\delta_{n}$.

Proof of Theorem 3. From the argument preceding Theorem 3 we see that it suffices to prove the equivalence of (2.11) and the condition,

$$
x^{p+1}\left[E\left\{X^{2} I(|X|>x)\right\}+x^{-2} E\left\{X^{4} I(|X| \leqslant x)\right\}\right] \rightarrow 0
$$

as $x \rightarrow \infty$. Two integrations by parts show that the left side of (3.17) equals

$$
l(x)=2 x^{p+1} \int_{x}^{\infty} u P(|X|>u) d u+4 x^{p-1} \int_{0}^{x} u^{3} P(|X|>u) d u .
$$

If $x^{p+3} P(|X|>x)<\varepsilon$ for all $x>\lambda$ then for such values of $x$,

$$
\begin{aligned}
l(x) & \leqslant 2 \varepsilon x^{p+1} \int_{x}^{\infty} u^{-p-2} d u+4 x^{p-1} \int_{0}^{\lambda} u^{3} d u+4 \varepsilon x^{p-1} \int_{\lambda}^{x} u^{-p} d u \\
& \rightarrow 2 \varepsilon /(1+p)+4 \varepsilon /(1-p)
\end{aligned}
$$

as $x \rightarrow \infty$. Therefore (2.11) implies (3.17). Conversely, since

$$
0 \leqslant x^{p+3} P(|X|>x) \leqslant x^{p+1} E\left\{X^{2} I(|X|>x)\right\},
$$

then (3.17) implies (2.11).

Proof of TheOREM 6. A portion of our proof closely resembles that of Theorem 5 of [7], and other parts are similar to the proof of Theorem 1 above. We omit many details. Define

$$
H_{n}(x)=P\left(S_{n} \leqslant n^{1 / 2} x\right)-\sum_{j=0}^{2 k-2} n^{-j / 2} P_{j}(-\Phi)(x),
$$


and let

$$
h_{n}(t)=\alpha^{n}\left(t / n^{1 / 2}\right)-\sum_{j=0}^{2 k-2} n^{-j / 2} P_{j}(i t) e^{-t^{2} / 2}
$$

denote the Fourier-Stieltjes transform of $H_{n}$. The argument preceding equation (3.7) in [7] shows that

$$
\int_{-\infty}^{\infty}|x|^{p} d H_{n}(x)=-K_{p} \int_{0}^{\infty} t^{-(p+1)} \operatorname{Re} h_{n}(t) d t .
$$

The argument preceding (3.10) in [7] gives

$$
\operatorname{Re} h_{n}(t)=n \gamma\left(t / n^{1 / 2}\right) e^{-t^{2} / 2}+r_{n 4}(t),
$$

where $\gamma(t)=o\left(t^{2 k}\right)$ as $t \rightarrow 0$, and for a sufficiently small $\varepsilon>0$ and $|t| \leqslant \varepsilon n^{1 / 2}$, $\left|r_{n 4}(t)\right| \leqslant o\left(n^{1-k}\right) t^{2 k+2} e^{-t^{2} / 5}$. We may deduce from (3.19) that

$$
\int_{0}^{\varepsilon n^{1 / 2}} t^{-(p+1)} \operatorname{Re} h_{n}(t) d t=o\left(n^{1-k}\right)
$$

as $n \rightarrow \infty$. An analogue of the argument following (3.10) in the proof of our Theorem 1 leads us to conclude that for any $\varepsilon \in(0, \pi / d]$,

$$
\begin{aligned}
J_{n} & \equiv \int_{\varepsilon n^{1 / 2}}^{\infty} \operatorname{Re} h_{n}(t) d t \\
& =\sum_{j=1}^{\infty} \int_{-\varepsilon n^{1 / 2}}^{\varepsilon n^{1 / 2}}\left(t+2 j \pi n^{1 / 2} / d\right)^{-(p+1)} \operatorname{Re} \alpha^{n}\left(t / n^{1 / 2}\right) d t+o\left(n^{1-k}\right) .
\end{aligned}
$$

However, it follows from (3.19) that

$$
\sum_{j=1}^{\infty} \int_{-\varepsilon n^{1 / 2}}^{\varepsilon^{1 / 2}}\left(t+2 j \pi n^{1 / 2} / d\right)^{-(p+1)} \operatorname{Re} h_{n}(t) d t=o\left(n^{1-k}\right),
$$

and consequently, since odd numbered $P_{j}$ 's are odd polynomials and even numbered $P_{j}$ 's are even polynomials,

$$
\begin{aligned}
J_{n}= & \sum_{j=1}^{\infty} \int_{-\varepsilon n^{1 / 2}}^{\varepsilon n^{1 / 2}}\left(t+2 j \pi n^{1 / 2} / d\right)^{-(p+1)}\left\{\sum_{l=0}^{k-1} n^{-l} P_{2 l}(i t)\right\} e^{-t^{2} / 2} d t+o\left(n^{1-k}\right) \\
= & \sum_{j=1}^{\infty} \sum_{l=0}^{k-1} \sum_{m=0}^{2 k-1} n^{-l}\left(\begin{array}{c}
-(p+1) \\
m
\end{array}\right) \int_{-\varepsilon n^{1 / 2}}^{\varepsilon n^{1 / 2}}\left(2 j \pi n^{1 / 2} / d\right)^{-(p+1)}\left(t d / 2 j \pi n^{1 / 2}\right)^{m} \\
& \times P_{2 l}(i t) e^{-t^{2} / 2} d t+o\left(n^{1-k}\right) \\
= & \sum_{l=0}^{k-1} \sum_{m=0}^{2 k-1} n^{-l-(1 / 2)(p+m+1)}\left(\sum_{j=1}^{\infty} j^{-(p+m+1)}\right)\left(\begin{array}{c}
-(p+1) \\
m
\end{array}\right)(d / 2 \pi)^{p+m+1} \\
& \times \int_{-\infty}^{\infty} t^{m} P_{2 l}(i t) e^{-t^{2} / 2} d t+o\left(n^{1-k}\right) .
\end{aligned}
$$

The last-written integral vanishes if $m$ is odd, and so we need only consider even $m$. Note that $l+\frac{1}{2}(p+2 m+1)>k-1$ if $m>\left[k-l-1-\frac{1}{2}(p+1)\right]$, and that $k-l-1-\frac{1}{2}(p+1)<0$ if $l>\left[k-1-\frac{1}{2}(p+1)\right]$. The proof of Theorem 6 is now easily completed. 


\section{REFERENCES}

1. B. von Bahr, On the convergence of moments in the central limit theorem, Ann. Math. Statist. 36 (1965), 808-818.

2. S. Bernstein, Quelques remarques sur le théorème limite Liapounoff, Dokl. Akad. Nauk SSSR (Comptes rendus) 24 (1939), 3-8.

3. R. N. Bhattacharya and R. Ranga Rao, Normal approximation and asymptotic expansions, Wiley, New York, 1976.

4. I. M. Gel'fand and G. E. Shilov, Generalized functions, vol. 1, Academic Press, New York, 1964.

5. F. Götze and C. Hippe, Asymptotic expansions in the central limit theorem under moment conditions, $\mathrm{Z}$. Wahrsch. Verw. Gebiete 42 (1978), 67-87.

6. P. Hall, On the rate of convergence of moments in the central limit theorem, J. Austral. Math. Soc. 25 (1978), 250-256.

7. Bounds on the rate of convergence of moments in the central limit theorem, Ann. Probab. 10 (1982), 1004-1018.

Department of Statistics, The faculties, Australian National University, P.O. Box 4, Canberra ACT 2600, Australia 